\title{
lon temperature gradient driven mode in presence of transverse velocity shear in magnetized plasmas
}

\section{Chakrabarti, N.; Juul Rasmussen, J.; Michelsen, Poul}

\section{Published in:}

Physics of Plasmas

Link to article, DOI:

$10.1063 / 1.1943867$

Publication date:

2005

Document Version

Publisher's PDF, also known as Version of record

Link back to DTU Orbit

Citation (APA):

Chakrabarti, N., Juul Rasmussen, J., \& Michelsen, P. (2005). lon temperature gradient driven mode in presence of transverse velocity shear in magnetized plasmas. Physics of Plasmas, 12(7), 074501.

https://doi.org/10.1063/1.1943867

\section{General rights}

Copyright and moral rights for the publications made accessible in the public portal are retained by the authors and/or other copyright owners and it is a condition of accessing publications that users recognise and abide by the legal requirements associated with these rights.

- Users may download and print one copy of any publication from the public portal for the purpose of private study or research.

- You may not further distribute the material or use it for any profit-making activity or commercial gain

- You may freely distribute the URL identifying the publication in the public portal 


\title{
Ion temperature gradient driven mode in presence of transverse velocity-shear in magnetized plasmas
}

\author{
Nikhil Chakrabarti \\ Saha Institute of Nuclear Physics, 1/AF Bidhannagar Calcutta - 700 064, India. \\ Jens Juul Rasmussen and Poul Michelsen \\ Association EURATOM-Riso National Laboratory, \\ Optics and Fluid Dynamics Department Riso P.O. Box 49, DK 4000 Roskilde, Denmark.

\begin{abstract}
The effect of sheared poloidal flow on the toroidal branch of the ion temperature gradient driven mode of magnetized nonuniform plasma is studied. A novel 'nonmodal' calculation is used to analyse the problem. It is shown that the transverse shear flow considerably reduced the growth of the instability. A small but finite amount of viscosity and/or diffusion enhanced the stabilization process.
\end{abstract}


One of the major problems in magnetically confined plasmas is the anomaly of particle and heat transport by plasma convection across the magnetic field ${ }^{1}$. This convection is generically driven by the free energy release during the growth of small scale fluctuations. Magnetic perturbation is one of the very dangerous perturbations which can cause complete disruption of the plasma confinement. Therefore, the design philosophy of fusion devices is to try to minimize such perturbations. There is experimental evidence ${ }^{2}$ that indeed, in the plasma edge [SOL (scrape-off-layer)] such perturbations are normally absent and plasma convection across a strong confining magnetic field is mainly controlled by electrostatic field and pressure gradient forces. In such a case, the plasma dynamics is well described by various drift wave $\operatorname{models}^{3,4}$.

Several experiments ${ }^{2,5,6}$ have revealed the existence of the sheared poloidal flows and associated radial electric field in the edge region of tokamak plasmas. Such flows are of general interest in connection with confinement and transport ${ }^{7}$. Analytical as well as numerical investigations were performed to explain the shear flow dynamics. Linear and nonlinear calculations $^{8,9}$ predict that unstable modes in tokamaks may be stabilized by a critical shear flow. Also, fully developed turbulence nonlinear analysis predicts that the turbulence level can be suppressed in the tokamak edge by the shear flow. Therefore, an important issue that must be addressed is whether this shear flow has significant effects on the linear Ion Temperature Gradient (ITG) mode also. In this paper, we consider some of the issues regarding the effect of such shear flows on the ITG mode.

Analytical studies of the ITG mode with shear-flow have been done by a standard 'modal approach'. In this traditional method perturbed quantities are expanded in Fourier integrals. The modes are assumed to have an exponential time behavior and if the imaginary part of the frequency is positive, the modes will grow in time, resulting in an instability. This method is well known and effective to solve problems involving the Hermitian operator which gives orthogonal eigenfunctions. But the operator with shear-flow is generally non Hermitian and the eigenfunctions are not orthogonal to each other. Detailed discussion of this was recently given by Tatsuno et al. ${ }^{10}$ Therefore, according to linear algebraic theory, a linear system may be amplified by arbitrary large factors even if the imaginary parts of all the eigenfrequencies are negative. It turns out that by using the common Fourier or Laplace method in a system with shear-flow we may overlook the secular and transient behavior of the mode, which can lead to an incorrect result even in the linear regime. In this paper, 
we use the 'non-modal' approach to solve for ion temperature gradient driven drift waves in presence of transverse shear-flow. The method, originally proposed by Kelvin, became popular recently and has been extensively used in a variety of problems. In this method, one considers the temporal evolution of spatial Fourier harmonics of the perturbations without any spectral expansion in time. The wave number of each spatial Fourier harmonic along the shear flow direction varies in time. This method is useful to analyze various physical phenomena involving shear-flow which describe the energy exchange between shear-flow and the perturbations. The ion temperature gradient driven drift wave has been widely studied in plasmas ${ }^{11}$. Numerical simulations and analytical work in this area suggest that a radial electric field is generated due to turbulent fluctuations, which in turn generates poloidal flow and consequently reduces the growth rate of fluctuations. In this Brief Communication we have performed a model calculation to show the influence of equilibrium shear flow on the ITG mode. Our calculation demonstrates that shear-flow has an importance influence on the ITG mode when certain conditions are satisfied.

In the present work we have studied the effect of velocity shear on the toroidal branch of ITG modes. There exist two different branches of ion temperature gradient driven modes. One of them, in which the ITG mode is coupled with the ion acoustic waves, is destabilized by the local temperature gradient, which is a "slab type". The other is destabilized by the bad curvature of the magnetic field lines in the presence of finite ion temperature gradient and is called the interchange type. This is also referred to as the "toroidal branch" of the ITG mode $^{12}$. Here we are focussing our attention on the latter type. First, we specifically examine the influence of sheared flows (perpendicular to the external magnetic field) on the toroidal branch of the linear ITG instability by the non modal method. Results indicate that velocity shear can reduce the growth of the fluctuations. We must emphasize that velocity shear alone cannot completely saturate the linear ITG instability, but can reduce the growth rate of the fluctuations. For complete stabilization of the linear ITG instability it is necessary to incorporate terms representing viscosity and/or diffusion in the model. In the present work, we include the contributions of both viscosity and diffusion and demonstrate the need for these effects for the complete stabilization of the linear ITG mode.

The article is organized as follows: First, the general equations for the toroidal ITG mode with the effect of velocity shear are formulated, then we analyze the stability of the linear ITG mode in presence of velocity shear by the nonmodal method. Numerical studies for 
the evolution of the ITG mode with shear flow are discussed and numerical solutions are displayed. Finally,we summarize the main results.

For the description of Ion Temperature Gradient (ITG) driven mode we consider a collisionless plasma in a sheared external magnetic field. The motion of ions is governed by the hydrodynamic equations in a magnetic field $\mathbf{B}=B \hat{e}_{z}$ and the electrons are assumed to satisfy the Boltzmann relation $n_{e}=n_{0}(x) \exp \left(e \phi / T_{e}\right)$. The dynamics is described in a two dimensional slab geometry. For simplicity we introduce a local slab co ordinate system with $\hat{R} \rightarrow \hat{x}, \hat{Z} \rightarrow \hat{y}$ and $-\hat{\zeta} \rightarrow \hat{z}$. This is a reasonable choice if the scalelengths of the instability and vortex structures of interest are much smaller than $R$, the major radius of the torus (and also $a$ the minor radius). Here we also assume that the mean quantities are functions of $x$, with the spatial length scale defined as $\left|L_{n}\right|^{-1}=d \ln T_{i 0}(x) / d x$. The equilibrium potential profile generating the velocity shear may be written as:

$$
\phi_{e q}(x)=v_{\perp 0}\left(x-x_{\perp 0}\right)+v_{\perp 0}^{\prime} \frac{\left(x-x_{\perp 0}\right)^{2}}{2 !}+v_{\perp 0}^{\prime \prime} \frac{\left(x-x_{\perp 0}\right)^{3}}{3 !}+\cdots
$$

It may be noted that typically the higher order derivatives in the Taylor expansion of $\phi_{e q}(x)$ are significantly smaller than the first order derivative. Therefore, the equilibrium potential profile may be approximated as $\phi_{e q}(x) \approx v_{\perp 0}\left(x-x_{\perp 0}\right)+v_{\perp 0}^{\prime}\left(x-x_{\perp 0}\right)^{2} / 2$. In the right hand side (RHS) of the above equation the first term indicates rigid rotation and the next term introduces inhomogeneity in the equilibrium flow, $x_{\perp 0}$ is the position where the plasma rotates rigidly. Next we made a further important assumption that the mode width is much smaller than the velocity profile scale length, so that the velocity is well represented by the first two terms of its Taylor series. It is also important to note that we have considered the potential profile such that it only describes linearly varying perpendicular flows, i.e. $v_{\perp 0}^{\prime}=0$. If $v_{\perp 0}^{\prime \prime} \neq 0$ the possibility of the Kelvin-Helmholtz instability must be considered.

A closed system of three equations, describing the ITG mode vortex in terms of the electrostatic potential $\phi$ and the ion pressure $p$ can be obtained as follows: First we combine the ion continuity equation with the quasi-neutrality condition (the perpendicular motion of ions is described by $\mathbf{E} \times \mathbf{B}$ and the polarization drift velocities) to obtain an equation for $\phi$, and the system is then completed by writing the equation for pressure $p$.

The following set of nonlinear fluid equations are obtained that describe the mode dynamics $^{3}$.

$$
\frac{d}{d t}\left(\phi-\nabla_{\perp}^{2} \phi\right)=-\left(1-2 \epsilon_{n}+K \nabla_{\perp}^{2}\right) \frac{\partial \phi}{\partial y}+2 \epsilon_{n} \frac{\partial p}{\partial y}+D \nabla_{\perp}^{2} \phi-\mu \nabla_{\perp}^{2} \nabla_{\perp}^{2} \phi,
$$




$$
\frac{d p}{d t}=-K \frac{\partial \phi}{\partial y}+D \nabla_{\perp}^{2} p
$$

where $d / d t \equiv\left(\partial / \partial t+\mathbf{V}_{\perp} \cdot \nabla\right)$. The velocity components in the convective derivative are given by $\mathbf{V}_{\perp}=\hat{e}_{z} \times \nabla\left[\phi_{e q}(x)+\phi\right]$. In the above equations $\phi$ and $p$ represent the perturbation amplitudes.

The normalization used in the equations above are as follows: the coordinate variables perpendicular to the magnetic field $B \hat{e}_{z}$, i.e., $x$ and $y$, are normalized by $\rho_{s}$, the ion Larmor radius at electron temperature (given by the ratio of sound speed to ion cyclotron frequency $\left.-c_{s} / \omega_{c i}\right)$. The variable $z$ is normalized by $L_{n}$ and time variable is normalized by $L_{n} / c_{s}$. All velocity components are normalized by $\rho_{s} c_{s} / L_{n}$ and their spatial derivatives are normalized by $c_{s} / L_{n}$. The total (equilibrium plus perturbed) potential is normalized by $T_{e} \rho_{s} / e L_{n}$ and the pressure by $p_{0} \rho_{s} / L_{n}$. The definitions of the parameters $K$ and $\epsilon_{n}$ are $K=\left(1+\eta_{i}\right)\left(T_{i} / T_{e}\right)$ where $\eta_{i}=L_{n} / L_{T}$ and $\epsilon_{n}=L_{n} / R$, where $\mathrm{R}$ is the radius of curvature of the magnetic field.

For cold ions and no toroidicity effect, the above set of equations are reduced to the well known Hasegawa-Mima equation. For $T_{i} \neq 0$, the coupled set of equations for $\phi$ and $p$ exhibits linear instability, which is often known as the 'toroidal branch' of the ITG mode' and the dispersion relation is

$$
\omega^{2}\left(1+k^{2}\right)-\omega k_{y}\left(1-2 \epsilon_{n}-K k^{2}\right)+2 k_{y}^{2} K \epsilon_{n}=0
$$

The condition for the instability is $K>0$, as discussed by Nycander et al. In the following we show that perpendicular velocity shear considerably reduces the growth of the fluctuations, while a small but finite dissipation (viscosity and diffusion) completely stabilizes the mode.

Now we demonstrate by a simple calculation how the ITG instability is suppressed by velocity shear. The basic nonlinear equations for this analysis with the inclusion of equilibrium flow, as mentioned before, may be written as

$$
\begin{aligned}
{\left[\frac{\partial}{\partial t}+v_{\perp 0}^{\prime}\left(x-x_{\perp}\right) \frac{\partial}{\partial y}+\hat{z} \times \nabla \phi \cdot \nabla\right]\left(\phi-\nabla_{\perp}^{2} \phi\right)+\left(1-2 \epsilon_{n}+K \nabla_{\perp}^{2}\right) \frac{\partial \phi}{\partial y}-2 \epsilon_{n} \frac{\partial p}{\partial y} } & =D \nabla_{\perp}^{2} \phi-\mu \nabla_{\perp}^{2} \nabla_{\perp}^{2} \phi \\
{\left[\frac{\partial}{\partial t}+v_{\perp 0}^{\prime}\left(x-x_{\perp}\right) \frac{\partial}{\partial y}+\hat{z} \times \nabla \phi \cdot \nabla\right] p+K \frac{\partial \phi}{\partial y} } & =D \nabla_{\perp}^{2} p
\end{aligned}
$$

where $x_{\perp}=x_{\perp 0}-v_{\perp 0} / v_{\perp 0}^{\prime}$.

In order to study the set of equations (4) and (5) it is very convenient to employ the scheme where a moving co-ordinate system is used and the temporal problem is examined directly. 
For this purpose we use a spatially-inhomogeneous Galilean transformation. Specifically we introduce a spatio-temporal co-ordinate transformation to the fixed reference frame $x-y$ to get the local moving frame (Lagrangian) going with mean shear flow,

$$
\xi=y-v_{\perp 0}^{\prime}\left(x-x_{\perp 0}\right) t \quad \text { and } \quad \tau=t .
$$

Equation (6) induces the following transformations of the partial derivatives:

$$
\partial_{x}=-v_{\perp 0}^{\prime} \tau \partial_{\xi}, \quad \partial_{y}=\partial_{\xi}, \quad \partial_{t}=\partial_{\tau}-v_{\perp 0}^{\prime}\left(x-x_{\perp 0}\right) \partial_{\xi},
$$

which effectively takes us from the laboratory to the local rest frame of the mean flow. In the new coordinate system, the initial inhomogeneity in space $\left(x-x_{\perp 0}\right)$ has been exchanged for a new inhomogeneity in time. With the help of these transformations and assuming solutions of the form $\phi=\phi(\xi, \tau) p=p(\xi, \tau)$, we obtain

$$
\begin{gathered}
\frac{\partial}{\partial \tau}\left[\left\{1-\left(1+v_{\perp 0}^{\prime 2} \tau^{2}\right) \frac{\partial^{2}}{\partial \xi^{2}}\right\} \phi\right]+\left[1-2 \epsilon_{n}+K\left(1+v_{\perp 0}^{\prime 2} \tau^{2}\right) \frac{\partial^{2}}{\partial \xi^{2}}\right] \frac{\partial \phi}{\partial \xi}-2 \epsilon_{n} \frac{\partial p}{\partial \xi} \\
=D\left(1+v_{\perp 0}^{\prime 2} \tau^{2}\right) \frac{\partial^{2} \phi}{\partial \xi^{2}}-\mu\left(1+v_{\perp 0}^{\prime 2} \tau^{2}\right) \frac{\partial^{2}}{\partial \xi^{2}}\left[\left(1+v_{\perp 0}^{\prime 2} \tau^{2}\right) \frac{\partial^{2} \phi}{\partial \xi^{2}}\right] \\
\frac{\partial p}{\partial \tau}+K \frac{\partial \phi}{\partial \xi}=D\left(1+v_{\perp 0}^{\prime 2} \tau^{2}\right) \frac{\partial^{2} p}{\partial \xi^{2}}
\end{gathered}
$$

We note that with the transformation used above the explicit $x$ dependence is eliminated from the equations, resulting in the vanishing convective nonlinear terms. This is expected since by this choice of co-ordinates we are reducing the dimensionality of the perturbations. Transformation of the partial derivatives shows that the shear flow term together with $\partial_{t}$ reduces to a simple 'temporal' derivative $\partial_{\tau}$. The set of equations $(7)$ and (8) is homogeneous with respect to $\xi$. Therefore, a Fourier transform of $\phi$ and $p$ with respect to $\xi$ yields a good quantum number $k_{\xi}$ and the equations may be solved by assuming the solution of the form $\psi(\xi, \tau)=\int d k_{\xi} \psi\left(k_{\xi}, \tau\right) \exp \left(i k_{\xi} \tau\right)$, where $\psi$ is either $\phi$ or $p$. With these solutions we find two coupled first order ordinary differential equations for $\phi$ and $p$. Finally the coupled system may be reduced to a second order differential equation in $\tau$. A numerical solution of these equations shows that $|\phi|,|p|$ are bounded for $v_{\perp 0}^{\prime} \rightarrow \infty$.

The equations can, however, be solved analytically in an approximate manner with a further assumption, $\mu \approx D$. Without any assumptions, here the system of partial differential equations (PDE's) (7) and (8) now become a system of ordinary differential equations 
(ODE's):

$$
\begin{gathered}
\frac{d}{d \tau}\left[\left(1+k_{\perp}^{2}\right) \phi\right]+\left(1-2 \epsilon_{n}-K k_{\perp}^{2}\right) i k_{\xi} \phi-2 \epsilon_{n} i k_{\xi} p=-D k_{\perp}^{2} \phi-\mu k_{\perp}^{2} k_{\perp}^{2} \phi \\
\frac{d p}{d \tau}+K i k_{\xi} p=-D k_{\perp}^{2} p
\end{gathered}
$$

where $k_{\perp}^{2}(\tau)=k_{\xi}^{2}\left(1+v_{\perp 0}^{\prime 2} \tau^{2}\right)$. Here the notation $k_{\perp}$ stands for the perpendicular wave vector and it is time dependent. Note that this time dependence enters through finite velocity shear. The dissipative terms $(D \approx \mu)$ in the above equations can be eliminated through further transformations $p=P \exp \left[-\mu \int k_{\perp}^{2}(\tau) d \tau\right]$ and $\phi=\left(\psi / 1+k_{\perp}^{2}\right) \exp \left[-\mu \int k_{\perp}^{2}(\tau) d \tau\right]$. The coupled differential equations for $\mathrm{P}$ and $\psi$ may then be combined to a second order ODE in $\tau$ as

$$
\frac{d^{2} \psi}{d \tau^{2}}+i k_{\xi} \frac{d}{d \tau}\left(\frac{1-2 \epsilon_{n}-K k_{\perp}^{2}}{1+k_{\perp}^{2}} \psi\right)-\frac{2 K \epsilon_{n} k_{\xi}^{2}}{1+k_{\perp}^{2}}=0 .
$$

For $v_{\perp 0}^{\prime}=0$, if we assume the temporal mode behavior as $\psi \sim \exp (-i \omega \tau)$ then from Eq. (11) we recover the local dispersion relation for the ITG instability. With finite $v_{\perp 0}^{\prime}$ we find that the equation is complicated and is solved numerically as an initial value problem using Matlab. However we analyze the Eq. (11) with some simplifying assumptions. We assume that the perpendicular wave vector $k_{\xi} \ll 1$, i.e., we are looking for the stability of the long perpendicular wavelength mode in the presence of transverse velocity shear. We use the transformation

$$
\psi=\Psi \exp \left(-\frac{1}{2} \int \frac{i k_{\xi}\left(1-2 \epsilon_{n}-K k_{\perp}^{2}\right)}{1+k_{\perp}^{2}} d \tau\right)
$$

in Eq. (11), and retaining terms up to $k_{\xi}^{2}$ and neglecting higher powers of $k_{\xi}$, we have the differential equation for $\Psi$ as

$$
\frac{d^{2} \Psi}{d \tau^{2}}+\left[\frac{k_{\xi}^{2}\left(1-2 \epsilon_{n}\right)^{2}}{\left(1+k_{\perp}^{2}\right)^{2}}-\frac{2 \epsilon_{n} k_{\xi}^{2} K}{1+k_{\perp}^{2}}\right] \Psi=0
$$

Clearly the second term in the square bracket is the destabilizing term for the ITG mode. To solve Eq. (13) analytically, we make an assumption of a long transverse ITG mode, i.e., $k_{\xi} \ll 1$. We neglect the term proportional to $k_{\xi}^{3}$ and retain the term $k_{\xi}^{2}$ in the numerator of the square bracketed second term. This assumption allows us to obtain the exact solution of equation (13) without any loss of generality.

Defining a new variable $\bar{\tau}=i k_{\xi} v_{\perp 0}^{\prime} \tau / \sqrt{1+k_{\xi}^{2}}$, we may express the above differential equation as

$$
\frac{d^{2} \Psi}{d \bar{\tau}^{2}}+\left[\frac{\alpha}{\left(1-\bar{\tau}^{2}\right)}-\frac{\beta}{\left(1-\bar{\tau}^{2}\right)^{2}}\right] \Psi=0
$$


where $\alpha=2 \epsilon_{n} K / v_{\perp 0}^{\prime 2}$ and $\beta=\left(1-2 \epsilon_{n}\right)^{2} / v_{\perp 0}^{\prime 2}\left(1+k_{\xi}^{2}\right)$. Equation (14) can be solved exactly and the solution may be expressed in terms of the associated Legendre functions,

$$
\psi(\tau)=\sqrt{\bar{\tau}^{2}-1}\left[A P_{\nu}^{m}(\bar{\tau})+B Q_{\nu}^{m}(\bar{\tau})\right]
$$

where $\nu=-1 / 2+\sqrt{1 / 4+\alpha}, m=\sqrt{1+\beta}, A$ and $B$ are two arbitrary constants. We see that our total solution $\Psi(\bar{\tau})$ consists of two linearly independent solutions, $\left|P_{\mu}^{m}(\bar{\tau})\right|$ which is unbounded, growing as $\tau^{\nu}$ (if $\nu$ is positive), while $\left|Q_{\nu}^{m}(\bar{\tau})\right|$ decays as $\tau^{-(\nu+1)}$. This implies that $\phi \sim \tau^{(\nu-1)} \exp \left[-\mu k_{\xi}^{2} \tau\left(1+v_{\perp 0}^{\prime 2} \tau^{2} / 3\right)\right.$. Therefore it is clear that in absence of dissipation, the solution still exhibits growth of the ITG mode if $\nu>1$. This explicitly means that the condition for the onset of instability is $v_{\perp 0}^{\prime}=\sqrt{\epsilon_{n} K}$. If the velocity shear parameter $v_{\perp 0}^{\prime}<\sqrt{\epsilon_{n} K}$, then the instability continues to grow algebraically; if $v_{\perp 0}^{\prime}$ exceeds this critical value, the ITG instability starts algebraically decaying. Since dissipation has little effect on long wavelength modes, we can only say that a finite value of dissipation can enhance the stabilization process.

In Eq. (15), the ratio $B / A$ is decided by the initial conditions at $\tau=0$. One may consider even $[\dot{\psi}(0)=0$, over-dot implies differentiation with respect to $\tau]$ and odd $[\psi(0)=0]$ solutions and determine $B / A$ in each case. As an example, we consider the even solution for which $(B / A)=-(2 / \pi) \sin [\pi(\nu+m) / 2] \exp [i \pi(3 m-\nu) / 2]$. In deriving the expression for $B / A$, only $\tau \geq 0$ is considered. We now proceed to calculate the amplification factor using the asymptotic form of the solutions (15) to write.

$$
\frac{\phi(\tau)}{\phi(0)}=F(\mu, m) \tau^{\nu-1}
$$

where

$$
F(\nu, m) \propto \frac{\sqrt{\pi}}{2^{1+m}} \frac{\Gamma\left(\nu+\frac{1}{2}\right) \Gamma 1+\frac{\nu-m}{2}}{\Gamma 1+(\nu-m) \Gamma \frac{1+\eta}{2}}\left[\cos \left(\frac{\pi \eta}{2}\right)+i \sin \left(\frac{\pi \eta}{2}\right) e^{i \pi(3 m-\nu)}\right]^{-1}
$$

and $\eta=\nu+m$. Note that even for $|F(\nu, m)| \sim 1$ for allowed values for $\nu, m$ we have a significant amount of amplification for the ITG waves. Therefore, our analysis indicates that for $v_{\perp 0}^{\prime} \neq 0$, long wavelength ITG modes are still unstable unless the shear flow amplitude reaches some critical value. Since $\tau$ is normalized to drift time scale $\left(c_{s} / L_{n}\right)$, we may regard $c_{s} / L_{n}$ as the typical growth rate for the instability.

Therefore, our specific contribution in this analytical calculation is that we have proposed a method which allows one to follow explicitly the interaction of shear flow with the linear 
ITG mode. The growth rate of the instability is calculated and the explicity solution for the amplification of the instability is presented.

Now we solve equations (9) and (10) numerically without any approximations. It has been assumed in the numerical solution of these equations that $|p|,|\phi| \ll 1$, although all the nonlinear terms from convective nonlinearity vanish exactly. This assumption may be justified, since we have shown that for finite velocity shear $\left(v_{\perp 0}^{\prime} \neq 0\right),|N|$ is bounded, unlike the solutions in the absence of velocity shear. For the numerical solution the standard ordinary differential equation solver from MATLAB is used. The results are presented for typical values for the shear flow parameter. In Figs. 1, 2 it is shown that potential and pressure fluctuations are growing without velocity shear and dissipative effects. A small amount of shear-flow starts reducing this growth of the fluctuations and for $v_{\perp 0}^{\prime}=0.5$ and $\mu=0.001 D=0.003$ fluctuations become stabilized. In both cases we have fixed the value of $\epsilon_{n}, K$ as $0.33,0.7$ respectively. Therefore, we conclude that shear flow has a huge impact on the reduction of the growth rate of the toroidal ITG instability.

In this work we have shown that the ion temperature gradient driven instability is considerably suppressed by shear flow in equilibrium. A recent paper ${ }^{11}$ has studied the effect of shear flow on the ITG instability with similar model equations without any dissipative mechanism. But the present manuscript has gone a step beyond introducing viscosity and diffusion in the basic equations and solved them analytically to demonstrate the importance of dissipation in complete stabilization of the mode. Dissipation $\mu \sim D$ has weak effect in the stability criterion, but it is essential for the compete saturation of the mode. There is a trivial difference between this paper and Ref. ${ }^{11}$. In the present manuscript the problem is solved without explicit $x$ dependence. Due to the reduction of the dimensionality of the perturbations, the $\mathbf{E} \times \mathbf{B}$ nonlinear term vanishes and the problem becomes linear and two dimensional. In Ref. ${ }^{11}$, the perturbations are linearized and include explicit $x$ dependence. It turns out that the problem studied in this manuscript consists essentially of two spatial dimensions, whereas in the ref. 11 it is three dimensional. It is important to mention that if one includes velocity curvature $\left(v_{\perp 0}^{\prime \prime}\right)$ in the equilibrium flow, then the Kelvin Helmhotz mode is likely to be unstable. As far as tokamak application is concerned, however, the influence of magnetic shear is considerably more significant than velocity curvature. Since magnetic shear has a further stabilizing effect on drift waves one should include this for direct tokamak application. However, this calculation shows that for the most unstable drift 
instability, velocity shear influences the growth rate of the mode.

It should be emphasized that the calculation presented in this paper does not consider the flow-fluctuation theory, i.e., it is not demonstrated how the flow profile changes and reacts back on the fluctuations self-consistently due to nonlinear effect.As mentioned in the introduction, our main objective in this paper is to show how the toroidal ITG mode is affected for a given equilibrium velocity shear. Deeper investigation is needed to examine the self consistent shear flow effect on the ITG mode in presence of magnetic shear.

1 K. H. Burrel, Phys. Plasmas 4, 1499 (1997).

2 S. J. Zweben, Phys. Fluids 28, 974 (1985).

3 B. G. Hong, F. Romanelli, and M. Ottaviani, Phys. Fluids B 3, 615 (1991).

4 S. Hamaguchi and W. Horton, Plasma Phys. Controlled Fusion 34, 203 (1992).

5 C. P. Ritz, R. D. Bengtson, S. J. Levinson, and E. J. Powers, Phys. Fluids. 27, 2956 (1984).

${ }^{6}$ G. R. Tynan, L. Schmitz, R. W. Conn, R. Doerner, and R. Lehmer, Phys.Rev. Letters 68, 3032 (1992).

7 J. D. Callen, Phys. Fluids B 4, 2142 (1992).

8 W. Horton, G. Hu, and G. Laval, Phys. Plasmas 3, 2912 (1996).

9 A. E. Koniges, J. A. Crotinger, and P. H. Diamond, Phys. Fluids B 4, 2785 (1992).

10 T. Tatsuno, V. Volponi, and Z. Yoshida, Phys. Plasmas 8, 399 (2001).

11 V. S. Mikhailenko and J. Weiland, Phys. Plasmas 9, 529 (2002).

12 N. Chakrabarti and J. J. Rasmussen, Phys. Plasmas 6, 3047 (1999). 


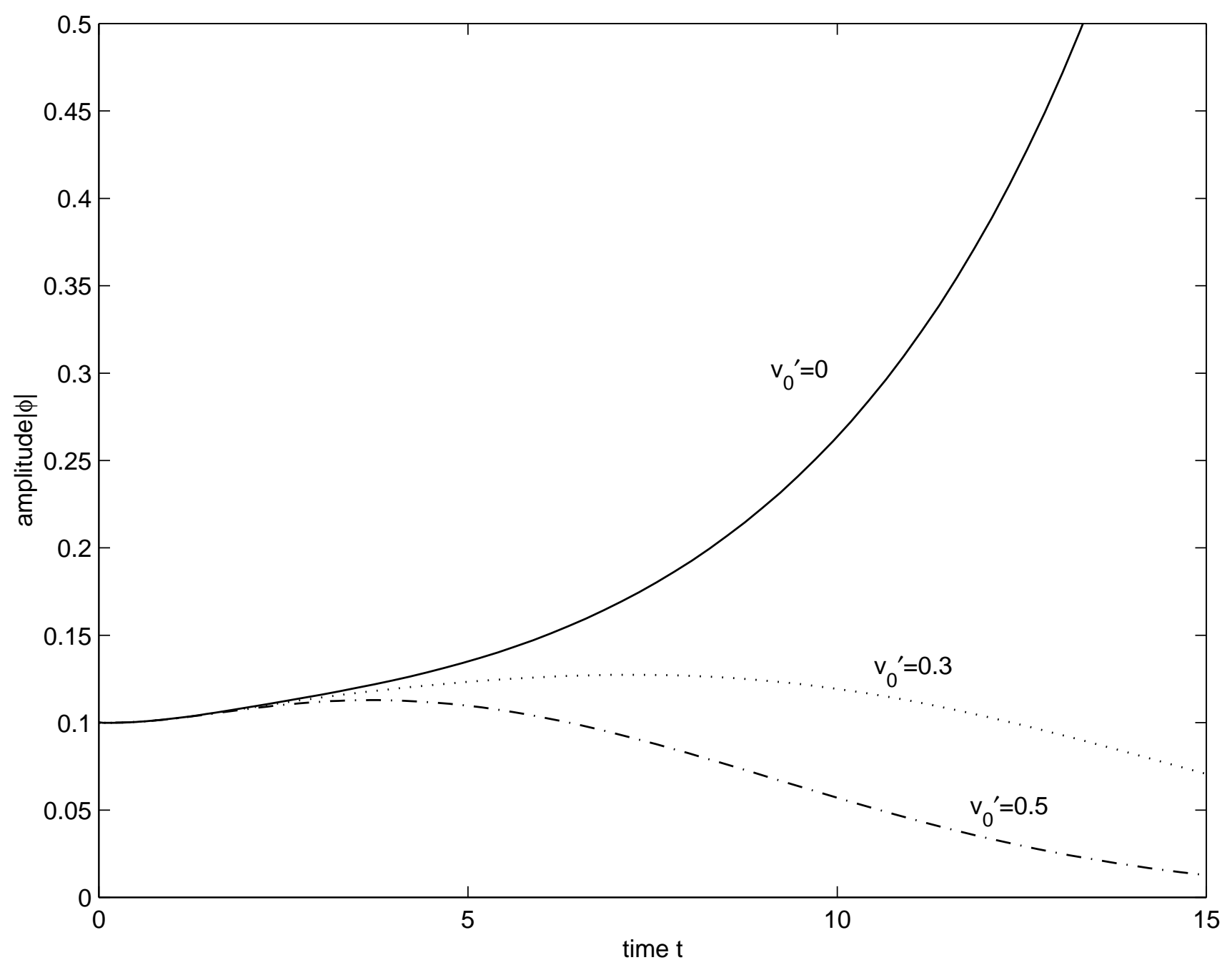

FIG. 1: Numerical solution of the ITG mode potential fluctuations for $k_{\xi}=0.6, \epsilon_{n}=0.33, K=0.7$, $\mu=0.001, D=0.003$, and different values of velocity shear parameter 


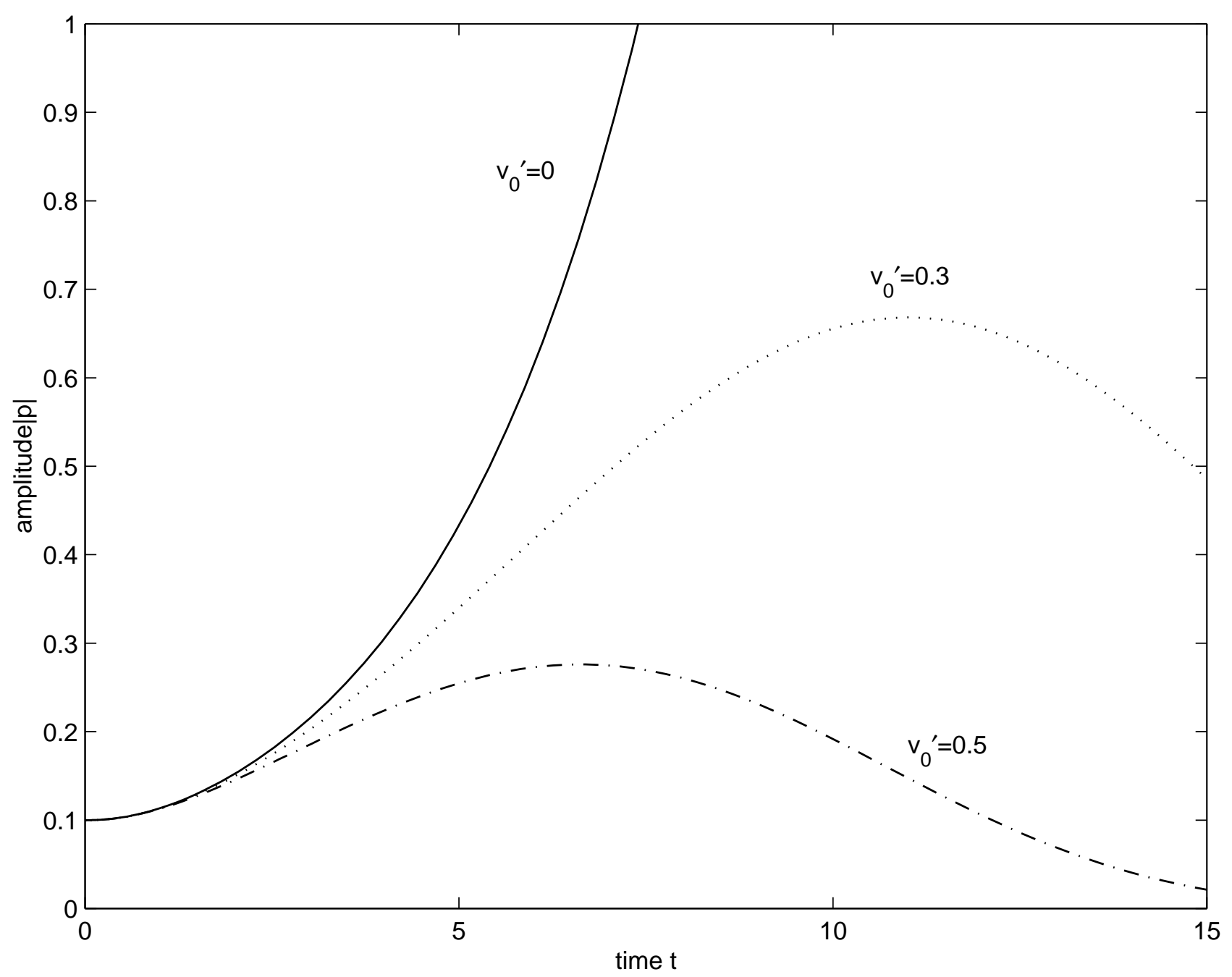

FIG. 2: Numerical solution of the ITG mode pressure fluctuations for $k_{\xi}=0.6, \epsilon_{n}=0.33, K=0.7$, $\mu=0.001, D=0.003$, and different values of velocity shear parameter 